\title{
Establishment on Information System Data Base of Provincial-level Farmland Quality Management
}

\author{
1,a Liu Tao 2,b Li Tingxuan ${ }^{3, c}$ Zheng Zicheng ${ }^{4, d}$ Wang Yongdong \\ 5,e Zhang Xizhou \\ $1,2,3,4,5$ College of resources and environment, Sichuan Agricultural Uniersity, Wenjiang, Sichuan \\ 611130, China \\ a,b,c,d,e environ2000@126.com
}

Keywords: Farmland quality; management and information system; data base.

\begin{abstract}
Data standardization and unification is the necessary condition of establishing basic data base of farmland base, if we want to use one data base, the same standard and model to manage farmland quality and data of each country as well as realize data sharing of farmland quality, we must use data standardization and unification.
\end{abstract}

\section{Introduction}

The standardization and unification of data is the necessary condition of establishing data base of farmland base, the basic data managed by this system is from each country, it must have uniform principle on data standardization. Farmland grade chart is the result on evaluation of farmland resources it also must have geographical element such as administrative division map, road and water area, so that it reflects the distribution condition of attribute data in each country. For standardization of attribute data, we should possibly use requirement of data dictionary of farmland land fertility evaluation, reduce work load of standard data, which is favorable for system generality.

\section{Data base of space}

Space data base is the storage place of GIS space data, design and realization of one space data base with rich content and reasonable structure is the key factor [2-8] of the whole system. This system is the 5-level information search system of province, city, country, town and village, of which provincial-level administrative division map and city-level administrative map is the foundation framework of space data, management of farmland quality uses country-level as basic unit, adopts search model of country, town and village, including farmland quality chart, country boundary map and village boundary map.

Space data base uses MS Sqlserver 2005 as closed basic data base, space data uses Krassowski eliipsoid of 1938 as parameter, Beijing 54 Coordinate System, 6 degree zone projection of Gauss Kruger, Yellow Sea altitude of 1956, and vector data is the shp form of data base of relationship type. 
Tab.1The Description of spatial data layers

\begin{tabular}{|c|c|c|c|c|}
\hline Name of layer & English name & Map pattern & Element type & Description \\
\hline province & province & Vector & Line & $\begin{array}{l}\text { Provincial-level } \\
\text { administrative } \\
\text { division map }\end{array}$ \\
\hline city & city & Vector & surface & $\begin{array}{l}\text { Provincial-level } \\
\text { administrative } \\
\text { division map }\end{array}$ \\
\hline county & county & Vector & surface & $\begin{array}{l}\text { Provincial-level } \\
\text { administrative } \\
\text { division map }\end{array}$ \\
\hline town & town & Vector & surface & $\begin{array}{l}\text { Provincial-level } \\
\text { administrative } \\
\text { division map }\end{array}$ \\
\hline village & village & Vector & surface & $\begin{array}{l}\text { Provincial-level } \\
\text { administrative } \\
\text { division map }\end{array}$ \\
\hline road & road & Vector & line & Road, railway \\
\hline water area & water area & Vector & Line or surface & River, swag \\
\hline farmland & farmland & Vector & surface & $\begin{array}{l}\text { Evaluation } \\
\text { outcome of soil } \\
\text { fertility }\end{array}$ \\
\hline
\end{tabular}

\section{Property data base}

Property data base is used to store all the farmland nutrient content, abundance and deficiency grade and quality grade of farmland quality etc. According to demand of management information of provincial-level farmland quality, it makes detailed analysis and design on property data in the system. Design of each data table is as follows:

(1)Provincial-level administrative division map(sys_province) is used to describe city ( state) and space position structure in Sichuan.(table 2).

Tab.2 The Provincial-level administrative divisions map attribute description

\begin{tabular}{llllll}
\hline Field name & $\begin{array}{l}\text { English } \\
\text { name }\end{array}$ & Data type & width & $\begin{array}{l}\text { decimal } \\
\text { place }\end{array}$ & description \\
\hline $\begin{array}{l}\text { Province } \\
\text { name }\end{array}$ & province & character & 20 & 0 & \\
$\begin{array}{l}\text { Boundary } \\
\text { type }\end{array}$ & Boundary & character & 2 & 0 & $\begin{array}{l}\text { Provincial-le } \\
\text { vel is 1 }\end{array}$ \\
\hline
\end{tabular}

(2) City-level administrative division map(sys_ city) is used to describe space and position structure of each country in province(table 3).

Tab.3 The City-level administrative divisions map attribute description

\begin{tabular}{llllll}
\hline Field name & $\begin{array}{l}\text { English } \\
\text { name }\end{array}$ & Data type & width & $\begin{array}{l}\text { decimal } \\
\text { place }\end{array}$ & description \\
\hline City name & city & character & 20 & 0 & City-level is \\
$\begin{array}{l}\text { Boundary } \\
\text { type }\end{array}$ & Boundary & character & 2 & 0 & 2 \\
$\begin{array}{l}\text { Province } \\
\text { name }\end{array}$ & province & character & 20 & 0 & \\
\hline
\end{tabular}


(3)Country-level administrative division map(sys_ county) is used to describe space and position structure of each province and city (table 4).

Tab.4 The Country-level administrative divisions map attribute description

\begin{tabular}{llllll}
\hline Field name & $\begin{array}{l}\text { English } \\
\text { name }\end{array}$ & Data type & width & $\begin{array}{l}\text { decimal } \\
\text { place }\end{array}$ & description \\
\hline $\begin{array}{l}\text { Country } \\
\text { name }\end{array}$ & county & character & 20 & 0 & \\
$\begin{array}{l}\text { Boundary } \\
\text { name }\end{array}$ & Boundary & character & 2 & 0 & $\begin{array}{l}\text { City-level is } \\
\begin{array}{l}\text { Province } \\
\text { name }\end{array}\end{array}$ \\
$\begin{array}{l}\text { City name } \\
\text { province }\end{array}$ & city & character & 20 & 0 & 2 \\
\hline
\end{tabular}

(4)Town-level administrative division map(sys_town) is used to describe space and position structure of each province, city and country(table 5).

Tab.5 The Rural locations in the map attribute description

\begin{tabular}{llllll}
\hline Field name & $\begin{array}{l}\text { English } \\
\text { name }\end{array}$ & Data type & width & $\begin{array}{l}\text { decimal } \\
\text { place }\end{array}$ & description \\
\hline Town name & town & Character & 20 & 0 & City-level is \\
$\begin{array}{l}\text { Boundary } \\
\text { type }\end{array}$ & Boundary & Character & 2 & 0 & 2 \\
$\begin{array}{l}\text { Province } \\
\text { name }\end{array}$ & province & Character & 20 & 0 & \\
$\begin{array}{l}\text { City name } \\
\text { Country }\end{array}$ & city & Character & 20 & 0 & \\
name & county & Character & 20 & & \\
\hline
\end{tabular}

(5)village administrative division map(sys_ village) is used to describe space and position structure of each province and city. (table 6).

Tab.6 The Location of the village community property description

\begin{tabular}{llllll}
\hline Field name & English name & Data type & width & $\begin{array}{l}\text { decimal } \\
\text { place }\end{array}$ & Description \\
\hline Village name & village & Character & 20 & 0 & City-level is \\
$\begin{array}{l}\text { Boundary } \\
\text { type }\end{array}$ & $\begin{array}{l}\text { Boundary } \\
\text { type }\end{array}$ & Character & 2 & 0 & 2 \\
$\begin{array}{l}\text { Province } \\
\text { name }\end{array}$ & province & Character & 20 & 0 & \\
$\begin{array}{l}\text { City name } \\
\text { Country }\end{array}$ & city & Character & 20 & 0 & \\
$\begin{array}{l}\text { name } \\
\text { Town name }\end{array}$ & town & Character & 20 & & \\
\hline
\end{tabular}

(6) Road(sys_road) is used to describe road structure of each province, city and country (table 7). 
Tab.7 The location of property described in the road

\begin{tabular}{llllll}
\hline Field name & $\begin{array}{l}\text { English } \\
\text { name }\end{array}$ & Data type & width & $\begin{array}{l}\text { decimal } \\
\text { place }\end{array}$ & Description \\
\hline Road name & & Character & 20 & 0 & \\
$\begin{array}{l}\text { Boundary } \\
\text { type }\end{array}$ & & Character & 2 & 0 \\
$\begin{array}{l}\text { Province } \\
\text { name } \\
\text { City name }\end{array}$ & city & Character & 20 & 0 \\
$\begin{array}{l}\text { Country } \\
\text { name }\end{array}$ & county & Character & 20 & 0 \\
\hline
\end{tabular}

(8) Water area(sys_ water area) is used to describe water area structure of each province, city and country (table 8).

Tab.8 The property description waters on map

\begin{tabular}{llllll}
\hline Field name & $\begin{array}{l}\text { English } \\
\text { name }\end{array}$ & Data type & width & $\begin{array}{l}\text { decimal } \\
\text { place }\end{array}$ & description \\
\hline $\begin{array}{l}\text { Water area } \\
\text { name }\end{array}$ & water area & Character & 20 & 0 \\
$\begin{array}{l}\text { Boundary } \\
\text { type }\end{array}$ & & Character & 2 & 0 \\
$\begin{array}{l}\text { Province } \\
\text { name }\end{array}$ & Character & 20 & 0 \\
$\begin{array}{l}\text { City name } \\
\text { Country }\end{array}$ & city & Character & 20 & 0 \\
name & County & Character & 20 & \\
\hline
\end{tabular}

(9) Farmland quality(sys_farmland), it is used to describe farmland position and property structure of each country, town and village(table 9). 
Tab.9 The land quality attributes described

\begin{tabular}{|c|c|c|c|c|c|}
\hline Field name & English name & Data type & Width & decimal place & Description \\
\hline Province name & province & Character & 20 & 0 & \\
\hline City name & city & Character & 20 & 0 & \\
\hline Country name & county & Character & 20 & & \\
\hline Town name & town & Character & 20 & 0 & \\
\hline Village name & village & Character & & & \\
\hline $\mathrm{pH}$ & $\mathrm{pH}$ & Number & 4 & 0 & PH value \\
\hline $\begin{array}{l}\text { effective } \\
\text { phosphorus }\end{array}$ & ap & Number & 4 & 1 & $\mathrm{mg} / \mathrm{kg}$ \\
\hline $\begin{array}{l}\text { available } \\
\text { potassium }\end{array}$ & ak & Number & 4 & 1 & $\mathrm{mg} / \mathrm{kg}$ \\
\hline organic matter & al & Number & 4 & 1 & $\mathrm{~g} / \mathrm{kg}$ \\
\hline total nitrogen & tn & Number & 4 & 1 & $\mathrm{~g} / \mathrm{kg}$ \\
\hline $\begin{array}{l}\text { alkali-hydrolyzable } \\
\text { nitrogen }\end{array}$ & $\mathrm{nt}$ & Number & 4 & 1 & $\mathrm{mg} / \mathrm{kg}$ \\
\hline $\mathrm{pH}$ grade & $\mathrm{pH} \mathrm{l}$ & Character & 20 & 0 & $\begin{array}{l}\text { Evaluation } \\
\text { grade }\end{array}$ \\
\hline $\begin{array}{l}\text { Grade of effective } \\
\text { phosphorus }\end{array}$ & apl & Character & 20 & 0 & $\begin{array}{l}\text { Evaluation } \\
\text { grade }\end{array}$ \\
\hline $\begin{array}{l}\text { Grade of available } \\
\text { potassium }\end{array}$ & akl & Character & 20 & 0 & $\begin{array}{l}\text { Evaluation } \\
\text { grade }\end{array}$ \\
\hline $\begin{array}{l}\text { Grade of organic } \\
\text { matter }\end{array}$ & aol & Character & 20 & 0 & $\begin{array}{l}\text { Evaluation } \\
\text { grade }\end{array}$ \\
\hline $\begin{array}{l}\text { Grade of total } \\
\text { nitrogen }\end{array}$ & tnl & Character & 20 & 0 & $\begin{array}{l}\text { Evaluation } \\
\text { grade }\end{array}$ \\
\hline $\begin{array}{l}\text { Grade of } \\
\text { alkali-hydrolyzable } \\
\text { nitrogen }\end{array}$ & ntl & Character & 20 & 0 & $\begin{array}{l}\text { Evaluation } \\
\text { grade }\end{array}$ \\
\hline square & area & Number & 8 & 1 & acre \\
\hline $\begin{array}{l}\text { Grade of soil } \\
\text { fertility }\end{array}$ & $\mathrm{f} 1$ & Number & 4 & 0 & $\begin{array}{l}\text { National } 10 \\
\text { grade }\end{array}$ \\
\hline
\end{tabular}

\section{Conclusion}

Data base is the core and foundation of system, including space data base and property data base. When making design of data base, we should use space data base as core, combine with property data base and model base, the program design idea faced with object. In the process, data in the data base should refer to content standard established by software engineering, which guarantees data sharing, information visit in provincial-level area, secondly, it requires consistence and completeness of data, it makes design according to object theory and observes standardization, completeness, expansion and practicability principle [9-14]of software engineering. 


\section{References}

[1] Li Guangmin. Grading Information Release System Study and Design of Farmland Based on WebGIS [D].[Thesis of master's degree].Wuhan of Hubei province,Huazhong Agricultural University,2006

[2] Li Ting,Zhu Tianbao. Design and Realization of Data base under Supermap Environment [J]. Railway science and engineering journal, 2007(1): 91-96.

[3] Zhang Yuandong. Search Design and WebGIS Realization of Light Space Data [D].[ Thesis of master's degree].Xian of Shanxi province,Northwest University,2009

[4] Liu Dong. Design and Realization on Digital Agriculture and Space Information Management Platform Based on WebGIS[D].[ Thesis of master's degree].Shanghai,East China Normal University,2006

[5] Cao Long. Design and Realization on GIS System of Geological Disaster Alert Based on Nerve Network [D].[ Thesis of master's degree].Xian of Shanxi province,ZhangXian university of electronic science and technology,2005

[6] Beijing Chaotu Geological Information and Technology Co., Ltd understand SuperMap Is .Net.

[7] Zhou Jian,Xu Dezhi. 3-layer Application Program Design Based on Web [J]. Journal of Xiangtan Teachers College (natural science version), 2008(1): 34-36.

[8] Cao Hui,Hou Zhuo. Design and Realization on Task Assessment Platform Based on ASP.NET 2.0 [J]. Software Magazine, 2009(12): 66-68.

[9] Xu Aimin,Lv Zhiping,Li Jian. Study on LBS System Based on J2EE Development Platform [J]. Mapping eport, 2004(3): 35-38.

[10] Chen Pengxiang. Design and Realization on Information System of Agricultural Resources Based on WebGIS [D].[ Thesis of master's degree].Wulumuqi of Xinjiang,Xinjiang Agriculture University,2009

[11] Hu Wenjie. Data Management and Application System Based on Network Soil Test Prescription [D].[ Thesis of master's degree].Wuhan of Hubei province,Huazhong Agricultural University,2009

[12] Ma Qingyuan. Digital Agriculture Study Based on Space and Information Technology [D]. [ Thesis of doctor's degree].Shanghai,East China Normal University,2007

[13] Zhu Jin. Design and Realization on Data Management System Based on Linux Geological Space [D].[ Thesis of master's degree],Hangzhou of Zhejiang province,Zhejiang University,2007

[14] Zhu Tianbao. Decision Model Study and System Development Based on GIS Road Line [D].[ Thesis of master's degree].Changsha of Hunan province,Institutes Of Technology Of Changsha,2006 